\title{
RELAXAMENTO COM IMAGEM GUIADA E PRESENÇA DE DEPRESSÃO EM PACIENTES COM CÂNCER DURANTE QUIMIOTERAPIA*
}

\author{
Adriana Cristina Nicolussi ${ }^{1}$, Namie Okino Sawada ${ }^{2}$, Fernanda Mara Coelho Cardozo ${ }^{3}$, Juliana Maria de Paula ${ }^{4}$
}

\begin{abstract}
RESUMO: O objetivo foi avaliar o efeito do Relaxamento com Imagem Guiada sobre a presença de depressão em pacientes com câncer durante quimioterapia. Estudo quase-experimental, realizado em duas centrais de quimioterapia, entre 2009-2011. O grupo intervenção foi submetido ao relaxamento com imagem guiada; os grupos intervenção $(n=73)$ e controle $(n=79)$ responderam ao Inventário de Depressão de Beck. Os cânceres mais frequentes foram: mama, intestinal e gástrico. Diferenças estatisticamente significantes $(p<0,05)$ entre os grupos (Teste de Mann-Whitney, $p=0,0158$ ) e entre os tempos (Teste de Wilcoxon, $p=0,0401$ ) foram encontradas, com o grupo intervenção apresentando mais pacientes "sem depressão" do que o controle. De acordo com Inventário de Depressão de Beck, os resultados evidenciaram que a intervenção ajudou a reduzir a presença de depressão nestes pacientes. Espera-se que a prática desta intervenção seja divulgada e difundida no trabalho do dia-a-dia do enfermeiro, para que, em um futuro próximo, os pacientes oncológicos possam se beneficiar com estas terapias.
\end{abstract}

DESCRITORES: Neoplasias; Quimioterapia; Depressão; Relaxamento; Imaginação; Terapias complementares.

\section{RELAXATION WITH GUIDED IMAGERY AND DEPRESSION IN PATIENTS WITH CANCER UNDERGOING CHEMOTHERAPY}

ABSTRACT: The present study aimed to assess the effect of relaxation and guided imagery on depression in cancer patients undergoing chemotherapy. Quasi-experimental study conducted in two chemotherapy centers between 2009-2011. The intervention group was subjected to relaxation with guided imagery; the intervention $(n=73)$ and control $(n=9)$ groups completed the Beck Depression Inventory. The most frequent cancers were breast, intestinal and gastric cancers. Statistically significant differences ( $p$ $<0.05$ ) were observed between the groups (Mann-Whitney test, $p=0.0158$ ) and times (Wilcoxon test, $p=0.0401$ ), and the intervention group had more patients "without depression" than the control group. According to Beck Depression Inventory, the results showed that intervention contributed to reduce the presence of depression among these patients. It is expected that this intervention be disseminated in routine nursing care, so that in the near future cancer patients may benefit from these therapies.

DESCRIPTORS: Neoplasias; Chemotherapy; Depression; Relaxation; Imagination; Complementary therapies.

\section{RELAJACIÓN CON IMAGEN GUIADA Y PRESENCIA DE DEPRESIÓN EN PACIENTES CON CÁNCER DURANTE QUIMIOTERAPIA}

RESUMEN: El propósito del estudio fue evaluar el efecto de la Relajación con Imagen Guiada sobre la depresión en pacientes con cáncer durante la quimioterapia. Estudio casi experimental, realizado en dos centrales de quimioterapia, entre 2009 y 2011 . El grupo de intervención fue sometido a la relajación con imagen guiada; los grupos de intervención ( $\mathrm{n}=73)$ y control ( $\mathrm{n}=79)$ contestaron al Inventario de Depresión de Beck. Los tipos de cáncer más frecuentes fueron: de mama, intestinal y gástrico. Hubo diferencias de estadística significantes $(p<0,05$ ) entre los grupos (Test de Mann-Whitney, $p=0,0158$ ) y entre los tiempos (Test de Wilcoxon, $p=0,0401$ ). El grupo de intervención presentó más pacientes "sin depresión" que el de control. De acuerdo con el Inventario de Depresión de Beck, los resultados evidenciaron que la intervención contribuyó con la reducción de la presencia de depresión en eses pacientes. Se considera importante que la práctica de esa intervención sea divulgada y difundida en el trabajo diario del enfermero, para que, en un futuro prójimo, los pacientes oncológicos puedan beneficiarse con esas terapias.

DESCRIPTORES: Neoplasias; Quimioterapia; Depresión; Relajación; Imaginación; Terapias complementarias.

\footnotetext{
*Artigo extraído da tese intitulada: "Relaxamento com imagem guiada: influência sobre a qualidade de vida relacionada à saúde de pacientes com câncer durante o tratamento quimioterápico". Escola de Enfermagem de Ribeirão Preto, Universidade de São Paulo, 2012.
}

${ }^{1}$ Enfermeira. Doutora em Ciências. Docente de Enfermagem da Universidade Federal do Triângulo Mineiro. Uberaba, MG, Brasil.

${ }^{2}$ Enfermeira. Doutora em Enfermagem. Docente da Escola de Enfermagem de Ribeirão Preto da Universidade de São Paulo. Ribeirão Preto, SP, Brasil.

${ }^{3}$ Enfermeira. Mestre em Enfermagem. Executiva de Vendas Becton Dickinson Brasil. Ribeirão Preto, SP, Brasil.

${ }^{4}$ Enfermeira. Doutoranda da Escola de Enfermagem de Ribeirão Preto da Universidade de São Paulo. Ribeirão Preto, SP, Brasil.

Autor Correspondente:

Adriana Cristina Nicolussi

Universidade Federal do Triângulo Mineiro

Pç. Manoel Terra, 330 - 38015-050 - Uberaba, MG, Brasil

Email: drinicolussi@yahoo.com.br
Recebido: 23/08/2016

Finalizado: 03/10/2016 


\section{INTRODUÇÃO}

O diagnóstico e tratamento do câncer é uma experiência altamente estressante para a maioria dos pacientes. Além do impacto emocional, os pacientes têm necessidade de tratamentos muito agressivos, como quimioterapia, radioterapia e cirurgia. Isto pode levar ao aparecimento de sintomas como dor, fadiga, distúrbio do sono, causar ansiedade e depressão, e consequentemente, afetar a Qualidade de Vida (QV) dos pacientes com câncer ${ }^{(1-3)}$.

Muitos pacientes por sofrerem estresse, manifestam sintomas depressivos em resposta a sua doença. Esta sintomatologia é em parte uma resposta à notícia do diagnóstico, pode diminuir com o tempo ou persistir por um longo período ${ }^{(4)}$.

Depressão é um transtorno mental comum e tem como principais sintomas: baixa autoestima, sentimento de culpa, humor deprimido, distúrbios do sono e apetite, perda de interesse, energia e concentração(5). Além destes, os pacientes enfrentam outras dificuldades como controle de sintomas da doença e/ou do tratamento, possível aumento do tempo de permanência hospitalar, baixa aderência ao tratamento e reduzido índice de sobrevivência ${ }^{(2-6)}$.

O impacto emocional do diagnóstico e o rigor do tratamento afetam de forma negativa a QV e isto pode persistir após o tratamento. As evidências demonstraram que variáveis psicossociais durante o diagnóstico e tratamento são preditores chaves de QV a curto e a longo-prazo e enfatiza a importância de uma intervenção psicossocial precoce a indivíduos diagnosticados com câncer ${ }^{(7-8)}$.

A quimioterapia pode causar efeitos colaterais indesejáveis, como imunidade diminuída para outras doenças, perda de cabelo, perda de apetite, náuseas, vômitos, fadiga, diminuição da libido ou impotência, bem como ansiedade e depressão ${ }^{(1,5)}$.

Atualmente, Terapias Complementares e Alternativas têm sido difundidas como uma prática no tratamento do paciente com câncer ${ }^{(6-7,9-11)}$, sendo as terapias mente-corpo uma das mais utilizadas, tais como: relaxamento, imagem guiada, meditação, yoga, hipnose, tai chi, grupos de apoio e espiritualidade.

Estas terapias baseiam-se no entendimento de que pensamentos, sentimentos, crenças e atitudes podem afetar e moldar todos os aspectos do funcionamento biológico. Elas reconhecem que tudo o que se realiza com o corpo físico pode modificar o funcionamento mental e psicológico ${ }^{(12-13)}$.

As terapias mente-corpo são usadas em sobreviventes de câncer para aumentar o relaxamento, reduzir estresse, ansiedade e dor, restabelecer o sono e melhorar o enfrentamento. Elas são bem apropriadas aos pacientes que enfrentam medo, estresse, ansiedade e incerteza. Estas terapias podem aumentar a habilidade do sobrevivente em enfrentar a doença e seus sintomas ${ }^{(2,9-10)}$.

Neste estudo foram utilizadas as terapias relaxamento e imagem guiada. O relaxamento leva a redução na excitação do sistema nervoso simpático, que marca a resposta de luta ou fuga e uma diminuição do nível de estresse. O relaxamento regular tem resultados impressionantes para pessoas com câncer, como diminuição dos níveis de estresse e aumento do funcionamento do sistema imunológico, diminuição da dor, menos efeitos colaterais da quimioterapia, diminuição da ansiedade, melhora do humor e menor supressão de emoções ${ }^{(12)}$.

A terapia imagem guiada envolve a imaginação para criar uma experiência sensorial e alcançar um objetivo clínico, que pode ser específico, como diminuir a frequência cardíaca, estimular a função imune ou reduzir a dor ou estresse, ou gerais, para promover o bem-estar físico e psíquico, sendo muitas vezes combinada com o relaxamento ${ }^{(14)}$.

Diante dessas considerações, esta pesquisa teve como objetivo avaliar o efeito da terapia complementar Relaxamento com Imagem Guiada sobre a presença de depressão em pacientes adultos com câncer durante o tratamento quimioterápico. Tendo como objetivos específicos: caracterizar a amostra inicial total e por grupo, identificar a presença de depressão, através do Inventário de Depressão de Beck (IDB) nos grupos - Grupo Controle (GC) e Grupo Intervenção (GI), em três tempos avaliados: Tempo1 (T1) - início da quimioterapia (linha de base), Tempo2 (T2) - após três meses e Tempo3 (T3) no final da pesquisa (após seis meses); e comparar o IDB entre os grupos e entre os tempos. 
Este é um estudo quantitativo, de delineamento quase-experimental - de grupo controle nãoequivalente anterior-posterior ${ }^{(15)}$.

Os critérios de inclusão foram: pacientes com idade superior a 18 anos, de ambos os sexos, diagnosticados com câncer iniciando tratamento quimioterápico, atendidos pelo Sistema Único de Saúde (SUS), no período de maio/2009 a dezembro/2011. Foram excluídos pacientes que tinham alguma dificuldade de compreensão ao responder perguntas simples.

A pesquisa foi realizada no Centro Especializado de Oncologia (CEON) do Hospital Sociedade Portuguesa de Beneficência e na Central de Quimioterapia do Hospital das Clínicas da Faculdade de Medicina de Ribeirão Preto-Universidade de São Paulo (HC-FMRP-USP).

Foi realizado levantamento semanal dos pacientes que estavam iniciando tratamento quimioterápico e aqueles que preencheram os critérios de inclusão foram abordados pela pesquisadora, que explicou os objetivos e convidou os pacientes a participarem da pesquisa.

A amostragem foi proposital/intencional, nesta o pesquisador seleciona, propositalmente, sujeitos considerados típicos da população em questão(15), portanto os pacientes que aceitaram participar da pesquisa e receberem as sessões de relaxamento com imagem guiada entraram para o GI e os pacientes que não participaram da intervenção, mas concordaram em participar do estudo respondendo ao IDB constituíram o GC.

Os pacientes do Gl foram submetidos à terapia relaxamento com imagem guiada, conduzida por uma gravação em $\mathrm{CD}^{(16)}$, com duração de 15 minutos cada sessão. O CD inicialmente solicita ao paciente que se posicione confortavelmente, em seguida o conduz a realizar movimentos de respiração e relaxamento em diversas partes do corpo. Posteriormente, conduz a imaginar-se em um local onde se sinta seguro e relaxado, pede para imaginar o câncer e as células de defesa o destruindo, seu corpo se revigorando. O conduz a visualizar uma cena de algo de que gosta muito de fazer e que não podia por causa da doença e que curado poderá realizá-lo, criando uma expectativa positiva. Depois, pede ao paciente que agradeça por ter dado este tempo a si e a sua saúde, para que continue vivendo a vida, e para ir se movimentando e abrindo os olhos devagar para retornar ao ambiente.

A primeira sessão ocorreu no primeiro dia do tratamento e foi realizada junto à pesquisadora, que possui seis anos de experiência com a terapia. Ao término da sessão foi entregue uma cópia do CD ao GI, para realizarem em casa por pelo menos uma a três vezes na semana no decorrer do tratamento quimioterápico.

Para avaliar a presença de depressão foi utilizado o Inventário de Depressão de Beck (IDB) adaptado para Brasil ${ }^{(17)}$, ele é constituído por 21 itens que avaliam: humor deprimido, pessimismo, sensação de fracasso, perda de satisfação, sentimento de culpa, sensação de punição, auto-rancor, auto-acusação, ideação suicida, choro, irritabilidade, isolamento social, indecisão, imagem corporal alterada, inibição para trabalho, anormalidade do sono, fatigamento, perda de apetite, perda de peso, preocupações somáticas e perda de libido.

Neste questionário, o paciente escolhe a frase que melhor descreve como ele se sentiu na semana que passou, incluindo o dia atual (da entrevista). A escala é graduada com afirmações de zero a três, no qual zero é ausência do sintoma e três a presença máxima do sintoma. O escore mínimo é zero e o máximo é 63. A análise dos escores é realizada através da somatória, sendo o ponto de corte para "sem depressão" de zero a 15, para "disforia" de 16 a 20 e para "depressão" de 21 a 63. Este instrumento foi aplicado nos tempos T1, T2, T3 em ambos os grupos nos dois centros de quimioterapia.

Para análise dos dados, foi utilizado o programa STATA/SE versão 12.0. Foram calculadas as médias e desvio padrão para análise descritiva dos dados, empregado Teste Exato de Fisher, Teste de MannWhitney e Teste de Wilcoxon, para evidenciar diferenças significantes.

A pesquisa foi aprovada pelo Comitê de Ética em Pesquisa da Escola de Enfermagem de Ribeirão Preto - USP, protocolo n.1002/2009. 
A amostra inicial total no T1 foi composta por 152 pacientes, sendo 79 do GC e 73 do Gl. A Tabela 1 apresenta a frequência e percentagem das características sócio-demográficas da amostra total e por grupo.

Tabela 1 - Frequência e percentagem das características sócio-demográficas da amostra por grupo (Controle e Intervenção) e total (linha de base). Ribeirão Preto, SP, Brasil, 2009-2011

\begin{tabular}{|c|c|c|c|c|}
\hline & CARACTERÍSTICAS & $\begin{array}{c}\text { GC } \\
\mathbf{N}(\%) \\
\end{array}$ & $\begin{array}{c}\text { GI } \\
\mathbf{N}(\%) \\
\end{array}$ & $\begin{array}{l}\text { Total } \\
\mathrm{N}(\%) \\
\end{array}$ \\
\hline \multirow[t]{3}{*}{ Sexo } & Feminino & $40(50,63)$ & $45(61,64)$ & $85(55,92)$ \\
\hline & Masculino & $39(49,37)$ & $28(38,36)$ & $67(44,08)$ \\
\hline & Total & $79(100)$ & $73(100)$ & $152(100)$ \\
\hline \multirow[t]{5}{*}{ Faixa Etária } & 18 a 39 anos & $3(3,80)$ & $6(8,22)$ & $9(5,92)$ \\
\hline & 40 a 59 anos & $43(54,43)$ & $40(54,79)$ & $83(54,61)$ \\
\hline & 60 a 79 anos & $31(39,24)$ & $27(36,99)$ & $58(38,16)$ \\
\hline & 80 anos e acima & $2(2,53)$ & $0(0,00)$ & $2(1,32)$ \\
\hline & Total & $79(100)$ & $73(100)$ & $152(100)$ \\
\hline \multirow[t]{5}{*}{ Estado Civil } & Solteiro & $13(16,46)$ & $10(13,70)$ & $23(15,13)$ \\
\hline & Casado & $47(59,49)$ & $40(54,79)$ & $87(57,24)$ \\
\hline & Viúvo & $10(12,66)$ & $7(9,59)$ & $17(11,18)$ \\
\hline & Divorciado/ amasiado & $9(11,39)$ & $16(21,92)$ & $25(16,45)$ \\
\hline & Total & $79(100)$ & $73(100)$ & $152(100)$ \\
\hline \multirow{10}{*}{$\begin{array}{l}\text { Profissão/ } \\
\text { Ocupação }\end{array}$} & Aposentados & $20(25,32)$ & $15(20,55)$ & $35(23,03)$ \\
\hline & Donas de casa, do lar & $23(29,11)$ & $13(17,81)$ & $36(23,68)$ \\
\hline & $\begin{array}{l}\text { Trabalho informal (exemplo: faxineira, } \\
\text { pedreiro) }\end{array}$ & $19(24,05)$ & $12(16,44)$ & $31(20,41)$ \\
\hline & Vendedor, comerciante & $11(13,92)$ & $10(13,70)$ & $21(13,82)$ \\
\hline & Trabalho rural & $4(5,06)$ & $2(2,74)$ & $6(3,95)$ \\
\hline & Autônomo & $1(1,27)$ & $5(6,85)$ & $6(3,95)$ \\
\hline & Professor & $0(0,00)$ & $5(6,85)$ & $5(3,29)$ \\
\hline & Técnico e administrativo & $1(1,27)$ & $7(9,59)$ & $8(5,26)$ \\
\hline & Profissional de saúde & $0(0)$ & $4(5,48)$ & $4(2,63)$ \\
\hline & Total & $79(100)$ & $73(100)$ & $152(100)$ \\
\hline \multirow{4}{*}{$\begin{array}{l}\text { Cidade/ } \\
\text { Procedência }\end{array}$} & Ribeirão Preto & $39(49,37)$ & $41(56,16)$ & $80(52,63)$ \\
\hline & Região de Ribeirão Preto & $37(46,84)$ & $31(42,47)$ & $68(44,74)$ \\
\hline & Outras regiões & $3(3,80)$ & $1(1,37)$ & $4(2,63)$ \\
\hline & Total & $79(100)$ & $73(100)$ & $152(100)$ \\
\hline \multirow{4}{*}{$\begin{array}{l}\text { Nível de } \\
\text { escolaridade }\end{array}$} & $\leq$ Ensino Fundamental & $60(75,95)$ & $40(54,79)$ & $100(65,79)$ \\
\hline & $\leq$ Ensino Médio & $10(12,66)$ & $16(21,92)$ & $26(17,11)$ \\
\hline & $\leq$ Ensino Superior & $9(11,39)$ & $17(23,29)$ & $26(17,11)$ \\
\hline & Total & $79(100)$ & $73(100)$ & $152(100)$ \\
\hline \multirow[t]{6}{*}{ Religião } & Católico & $57(72,15)$ & $50(68,49)$ & $107(70,39)$ \\
\hline & Evangélico/cristão & $20(25,32)$ & $15(20,55)$ & $35(23,03)$ \\
\hline & Espírita & $1(1,27)$ & $5(6,85)$ & $6(3,95)$ \\
\hline & Ateu & $1(1,27)$ & $2(2,74)$ & $3(1,97)$ \\
\hline & Budista & $0(0,00)$ & $1(1,37)$ & $1(0,66)$ \\
\hline & Total & $79(100)$ & $73(100)$ & $152(100)$ \\
\hline
\end{tabular}

Fonte: Dados da pesquisa, 2012. GC= Grupo Controle; GI = Grupo Intervenção; n= frequência 
Conforme mostra a Tabela 1, a amostra inicial total foi predominantemente composta por mulheres, na faixa etária entre 40 e 59 anos, casadas, aposentadas ou donas de casa, católicas, residentes em Ribeirão Preto e com baixo nível de escolaridade (ensino fundamental).

A Tabela 2 apresenta a frequência e percentagem das características clínico-terapêuticas da amostra total e por grupo.

Tabela 2 - Frequência e percentagem das características clínico-terapêuticas da amostra por grupo (Controle e Intervenção) e total (linha de base). Ribeirão Preto, SP, Brasil, 2009-2011

\begin{tabular}{|c|c|c|c|c|}
\hline & CARACTERÍSTICAS & $\begin{array}{c}\text { GC } \\
\text { N (\%) }\end{array}$ & $\begin{array}{c}\text { GI } \\
\mathbf{N}(\%)\end{array}$ & $\begin{array}{l}\text { Total } \\
\text { N (\%) }\end{array}$ \\
\hline \multirow[t]{11}{*}{ Localização do câncer } & Mama & $12(15,19)$ & $24(32,88)$ & $36(23,68)$ \\
\hline & Gástrico & $13(16,46)$ & $6(8,22)$ & $19(12,50)$ \\
\hline & Intestinal & $20(25,32)$ & $12(16,44)$ & $32(21,05)$ \\
\hline & Ginecológico & $5(6,33)$ & $10(13,70)$ & $15(9,87)$ \\
\hline & Pulmão/mediastino & $9(11,39)$ & $6(8,22)$ & $15(9,87)$ \\
\hline & Geniturinário & $2(2,53)$ & $3(4,11)$ & $5(3,29)$ \\
\hline & Cabeça e pescoço & $9(11,39)$ & $4(5,48)$ & $13(8,55)$ \\
\hline & Neurológico & $4(5,06)$ & $1(1,37)$ & $5(3,29)$ \\
\hline & Leucemias/linfomas & $3(3,80)$ & $5(6,85)$ & $8(5,26)$ \\
\hline & Pele e melanomas & $2(2,53)$ & $2(2,74)$ & $4(2,63)$ \\
\hline & Total & $79(100)$ & $73(100)$ & $152(100)$ \\
\hline \multirow[t]{3}{*}{ Metástase } & Sim & $21(26,58)$ & $14(19,18)$ & $35(23,03)$ \\
\hline & Não & $58(73,42)$ & $59(80,82)$ & $117(76,97)$ \\
\hline & Total & $79(100)$ & $73(100)$ & $152(100)$ \\
\hline \multirow[t]{3}{*}{ Realização de cirurgia } & Sim & $59(74,68)$ & $58(79,45)$ & $117(76,97)$ \\
\hline & Não & $20(25,32)$ & $15(20,55)$ & $35(23,03)$ \\
\hline & Total & $79(100)$ & $73(100)$ & $152(100)$ \\
\hline \multirow[t]{3}{*}{ Realização de radioterapia } & Sim & $25(31,65)$ & $17(23,29)$ & $42(27,63)$ \\
\hline & Não & $54(68,35)$ & $56(76,71)$ & $110(72,37)$ \\
\hline & Total & $79(100)$ & $73(100)$ & $152(100)$ \\
\hline
\end{tabular}

Fonte: Dados da pesquisa, 2012. GC= Grupo Controle; GI = Grupo Intervenção; $n=$ frequência

De acordo com a Tabela 2, os cânceres mais frequentes encontrados foram: mama, intestinal e gástrico, a maioria dos pacientes havia realizado cirurgia e alguns deles também foram submetidos à radioterapia.

Quanto ao protocolo de quimioterapia, foi encontrado 46 esquemas diferentes de medicações, sendo os mais utilizados: 5-Fluorouracil + Leucovorin em 19 (12,50\%) pacientes; Cisplatina em 18 (11,84\%); Paclitaxel + Carboplatina em 17 (11,18\%); Oxaliplatina + Capecitabina (endovenosa e/ou via oral) em 12 (7,89\%); e Epirrubicina + Ciclofosfamida + Docetaxel em 12 (7,89\%) pacientes.

A maioria dos pacientes (76,97\%) relatou efeitos colaterais da quimioterapia, como: náuseas, vômitos, constipação, diarreia, mal-estar físico, fadiga, fraqueza, dor, insônia, perda de apetite, queixas de irritabilidade e ansiedade.

A Tabela 3 apresenta frequências e percentagens dos escores do IDB da amostra por grupo. Mostra também Teste Exato de Fischer para evidenciar diferenças de depressão entre os grupos nos três tempos, significância $\mathrm{p}<0,05$. 
Tabela 3 - Frequência e percentagem do Inventário de Depressão de Beck (T1, T2 e T3), Grupos Controle e Intervenção. Ribeirão Preto, SP, Brasil, 2009-2011

\begin{tabular}{|c|c|c|c|c|c|c|}
\hline \multirow[t]{2}{*}{ Escores } & GC & GC & GC & GI & GI & GI \\
\hline & N (\%)T1 & N (\%)T2 & N (\%)T3 & N (\%)T1 & N (\%)T2 & N (\%)T3 \\
\hline Sem Depressão & $63(80,77)$ & $40(65,57)$ & $29(60,42)$ & $52(71,23)$ & $48(82,76)$ & $39(86,67)$ \\
\hline Disforia & $9(11,54)$ & $13(21,31)$ & $14(29,17)$ & $13(17,81)$ & $5(8,62)$ & $2(4,44)$ \\
\hline Depressão & $6(7,69)$ & $8(13,11)$ & $5(10,42)$ & $8(10,96)$ & $5(8,52)$ & $4(8,89)$ \\
\hline Total & $78(100)$ & $61(100)$ & $48(100)$ & $73(100)$ & $58(100)$ & $45(100)$ \\
\hline
\end{tabular}

Fonte: Dados da pesquisa, 2012.

$\mathrm{GC}=$ Grupo Controle; GI = Grupo Intervenção; $\mathrm{n}=$ freqüência; $\mathrm{T} 1=$ Tempo 1; T2 = Tempo 2; T3 = Tempo 3.

Teste Exato de Fisher: T1 = 0,360, T2 = 0,086 e T3 = 0,004*

A última linha da Tabela 3 evidencia as perdas ocorridas nos grupos no decorrer do tempo. No GC houve perda de 17 pacientes de T1 para T2 e outros 13 pacientes para T3 e no GI, 15 e 13 pacientes de T1 para T2 e T3, respectivamente. Observou-se que, mesmo com estas perdas, a percentagem de pacientes sem depressão aumentou e a percentagem de pacientes com disforia e depressão diminuiu para o GI, enquanto que ocorreu o inverso para o GC.

A Tabela 4 apresenta Teste de Mann-Whitney do IDB entre os grupos Controle e Intervenção nos T1, T2 e T3.

Tabela 4 - Teste de Mann-Whitney do instrumento Inventário de Depressão de Beck (T1, T2 e T3), Grupos Controle e Intervenção. Ribeirão Preto, SP, Brasil, 2009-2011

\begin{tabular}{lcc} 
Variáveis - Grupo Controle/ Intervenção & z & p \\
\hline Inventário de Depressão de Beck T1 & $-1,827$ & 0,068 \\
\hline Inventário de Depressão de Beck T2 & 0,825 & 0,409 \\
\hline Inventário de Depressão de Beck T3 & 2,413 & $0,0158^{*}$
\end{tabular}

Fonte: Dados da pesquisa, 2012.

* $\mathrm{p}<0,05 ; \mathrm{z}=$ Teste de Mann-Whitney; T1 = Tempo 1; T2 = Tempo 2; T3 = Tempo 3.

Considerando significantes valores $\mathrm{p}<0,05$, os mesmos foram encontrados somente em T3, cujos pacientes "sem depressão" foram maiores para o GI do que para o GC, mesmo com as perdas ocorridas no decorrer do estudo.

A Tabela 5 apresenta Teste de Wilcoxon do instrumento IDB nos GC e GI, comparando os tempos T1xT2,T1xT3,T2xT3, considerando significantes valores $\mathrm{p}<0,05$.

As diferenças estatisticamente significantes foram encontradas de T1 para T2 e de T1 para T3 no GC, com resultados negativos e de $\mathrm{T} 1$ para $\mathrm{T} 3$ no GI com resultados positivos, indicando uma melhora para o GI em relação ao GC.

Tabela 5 - Teste de Wilcoxon do instrumento IDB (T1xT2, T1xT3 e T2xT3), Grupos Controle e Intervenção. Ribeirão Preto, SP, Brasil, 2009-2011

\begin{tabular}{lcccc} 
Variáveis & Grupo Controle & Grupo Controle & Grupo Intervenção & Grupo Intervenção \\
\cline { 2 - 5 } & $\mathbf{z}$ & $\mathbf{p}$ & $\mathbf{z}$ & $\mathbf{p}$ \\
\hline $\mathrm{IDB}(\mathrm{T} 1) \times \operatorname{IDB}(\mathrm{T} 2)$ & $-3,552$ & $0,0004^{*}$ & 0,349 & 0,727 \\
\hline $\mathrm{IDB}(\mathrm{T} 1) \times \operatorname{IDB}(\mathrm{T} 3)$ & $-2,922$ & $0,0035^{*}$ & 2,053 & $0,0401^{*}$ \\
\hline $\mathrm{IDB}(\mathrm{T} 2) \times \operatorname{IDB}(\mathrm{T} 3)$ & $-0,521$ & 0,602 & 1,371 & 0,171
\end{tabular}

Fonte: Dados da pesquisa, 2012.

* $\mathrm{p}<$ 0,05; $\mathrm{z}=$ Teste de Wilcoxon; IDB = Inventário de Depressão de Beck; T1 = Tempo 1; T2 = Tempo 2; T3 = Tempo 3. 


\section{- DISCUSSÃO}

As características sócio-demográficas e clínicas encontradas neste estudo foram semelhantes a outros estudos nacionais e internacionais ${ }^{(1,16,18)}$. Quanto aos tipos de câncer mais frequentes encontrados estão de acordo com as estimativas para o ano de 2011 (término da coleta de dados) e mantém-se semelhantes às estimativas para 2016 e 2017 no Brasil(19).

Com relação ao IDB, tanto para GC quanto para GI, a maioria dos pacientes estava "sem depressão" nos três momentos. Na comparação entre os grupos, as diferenças foram significantes somente em T3, com mais pacientes "sem depressão" no GI. Na comparação entre os tempos, as diferenças estatisticamente significantes encontradas para GC foram de T1 para T2 e de T1 para T3 e para o Gl de T1 para T3.

Estudo $^{(1)}$ examinou a eficácia de um programa de estratégia de enfrentamento composto por informações educacionais, reestruturação cognitiva, aprimoramento de habilidades de enfrentamento e relaxamento com imagem guiada e comparou com um grupo controle, em pacientes com câncer de mama em quimioterapia. Os resultados em um ano de seguimento mostraram que o GI $(n=38)$ teve melhora significante na QV geral, no bem-estar psicológico/espiritual $(p<0,01)$, na saúde, bemestar funcional e bem-estar socioeconômico $(p<0,05)$ comparado com o $G C(n=35)$. O GC reportou depressão leve de acordo com o IDB, porem não houve diferença estatisticamente significante nos escores de depressão entre os grupos.

Pesquisa ${ }^{(3)}$ avaliou resultados relativos a padrões de regulação emocional, percepção e satisfação com suporte social após intervenção cognitivo-comportamental em gestão do estresse que constituiu em relaxamento muscular, reestruturação cognitiva e treino em estratégias de enfrentamento e comparou com GC em pacientes com câncer em radioterapia com presença de fadiga no início e final do tratamento. O GI $(n=35)$ apresentou diminuição significativa $(p<0,001)$ da ansiedade, depressão e estresse, avaliada pela Escala de Ansiedade, Depressão e Stress, e aumento da percepção e satisfação com suporte social enquanto que o $\mathrm{GC}(\mathrm{n}=35)$ apresentou o inverso.

Estudo $^{(6)}$ examinou o efeito da musicoterapia e relaxamento muscular progressivo sobre a depressão, ansiedade e tempo de permanência hospitalar, em mulheres com câncer de mama após mastectomia radical, e comparou com GC que recebeu cuidados de rotina. A intervenção foi iniciada após 48 horas da cirurgia e realizada 2x/dia até a alta hospitalar. A análise mostrou que pacientes do GI $(\mathrm{n}=85)$ tiveram melhora significante na ansiedade $(\mathrm{p}<0,017)$, avaliada pelo State Anxiety Inventory (SAI), depressão $(p<0,001)$ avaliada pela Zung Self-rating Depression Scale (ZSDS) e tiveram menor tempo de permanência hospitalar do que o GC $(n=85)$ com significância estatística $(p<0,001)$.

O impacto da imagem guiada foi avaliado em pacientes submetidos à radioterapia para câncer de mama. As sessões da imagem guiada eram realizadas antes da radioterapia e as pacientes $(n=60)$ levaram CD para realizarem reforço em casa. Foi utilizado o questionário EuroQol Group's EQ-5D antes e após a sessão, que avalia cinco domínios de estado de saúde atual (mobilidade, auto-cuidado, atividades usuais, dor ou desconforto, ansiedade ou depressão), os resultados mostraram paradoxalmente aumento na dor e desconforto $(p<0,001)$ e diminuição nos níveis de ansiedade e depressão $(p=0,01)$. Estes resultados ilustraram o impacto positivo da imagem guiada e apóiam a incorporação de práticas alternativas à prática padrão(10).

Uma intervenção psicológica constituída por relaxamento e imagem guiada foi avaliada e comparada com um GC $(n=100)$. Os grupos foram pacientes com câncer de mama e de próstata em quimioterapia. No GI ( $n=100)$, coletaram saliva antes e após as sessões de relaxamento e imagem guiada e notaram que o nível de amilase salivar reduziu e reagiu mais rapidamente do que o cortisol salivar com a intervenção, sugerindo melhor relaxamento. A técnica de relaxamento com imagem guiada foi efetiva na redução dos níveis de ansiedade, avaliada pela Self-rating Anxiety Scale (SAS), depressão, através do IDB e desconforto corporal no GI ${ }^{(11)}$.

Estudo $^{(20)}$ testou a eficácia de seis meses de uma intervenção composta por uma hora de orientações fornecidas por enfermeira especialista em oncologia, um manual com informações sobre o tratamento quimioterápico, como efeitos adversos, exercício e dieta, um audiotape com 30 minutos de técnicas de relaxamento e quatro telefonemas mensais para acompanhamento feito pela enfermeira e comparou 
com GC, ambos os grupos constituídos por pacientes com câncer colorretal. Avaliaram auto-eficácia, sintomas de aflição, ansiedade, depressão (avaliados pelo Hospital Anxiety and Depression Scale HADS) e QV e encontraram que os pacientes no $\mathrm{GI}(\mathrm{n}=76)$ tiveram melhora significante na auto-eficácia $(p=0,003)$, redução na severidade dos sintomas $(p=0,01)$, interferência do sintoma $(p=0,025)$, ansiedade $(p=0,006)$ e depressão $(p=0,003)$, verificados aos três e seis meses, comparados com o GC ( $n=76)$, e sem diferença estatisticamente significante na QV.

Pesquisa que avaliou a frequência dos sintomas de depressão através do IDB em 41 pacientes com câncer de cabeça e pescoço em tratamento radioterápico encontrou que a disforia aumentou ao longo do tratamento, assim como o número de pacientes com depressão. Houve diferença estatisticamente significante entre a primeira e terceira aplicação do IDB, indicando que as mudanças dos sintomas de disforia e depressão foram significativas ao se considerar o início e o final do tratamento. Os resultados mostraram a importância dos profissionais de saúde detectarem a prevalência e os níveis dos sintomas de depressão, uma vez que esses sintomas tendem a aumentar podendo levar a consequências como falta de aderência ao tratamento e diminuição da QV desses pacientes ${ }^{(18)}$.

Com relação às perdas encontradas neste estudo, a literatura também demonstrou essas dificuldades ${ }^{(10,21)}$. Elas ocorreram por motivos como óbito de pacientes, complicações na saúde que os impediram de continuar no estudo, como por exemplo, acidente vascular cerebral com sequelas, por desistência e por término do tratamento quimioterápico antes do previsto, ou seja, antes do término da pesquisa.

Estudo $^{(21)}$ mostrou que a imagem guiada atraiu pacientes com altos níveis de ansiedade e baixo bem-estar emocional, porém falhou em reter os pacientes. Pacientes do grupo de imagem guiada que desistiram tiveram média do escore de bem-estar físico de 16,22 versus 19,61 ( $p=0,03$ ), de bem-estar emocional de 12,44 versus $17,1(p=0,07)$ e do escore total do FACT-G de 74,00 versus 85,85 ( $p=0,03)$ do que os participantes que permaneceram no estudo. Pacientes que desistiram dos grupos de intervenção tiveram mais severidade de sintomas, sintomas depressivos e ansiedade e pior bem-estar físico e emocional.

Nota-se que no Brasil ainda existe resistência e desconhecimento sobre as práticas complementares como relaxamento e imagem guiada, isso reflete nos poucos estudos publicados recentemente relacionados a esta temática, porém a literatura internacional encontrada demonstrou que o relaxamento com imagem guiada, usadas separadamente, juntas e/ou associadas a outras terapias, são utilizadas em pacientes com câncer em diferentes tratamentos, além da quimioterapia, como a radioterapia e cirurgia, sendo que a maioria demonstrou resultados positivos com o uso destas terapias.

\section{CONCLUSÃO}

Este estudo que avaliou o efeito da intervenção Relaxamento com Imagem Guiada sobre a presença de depressão em pacientes com câncer em tratamento quimioterápico possibilitou observar que a caracterização da amostra foi semelhante a outros estudos realizados no Brasil e no mundo e que a frequência encontrada dos tipos de cânceres neste estudo corrobora as incidências de câncer estimadas.

De acordo com o IDB, houve diferenças estatisticamente significantes entre os grupos e entre os tempos, com o GI apresentando mais pacientes "sem depressão" do que o GC, no final do estudo.

Como limitação, destaca-se a dificuldade em realizar um estudo quase-experimental, uma vez que depende da adesão do paciente para esse tipo de intervenção.

Esta pesquisa contribui para divulgação de abordagens mente-corpo como relaxamento e imagem guiada que podem ser realizadas pelo enfermeiro para prover uma redução na presença de depressão em pacientes com câncer em tratamento quimioterápico e também visando incentivar novos estudos sobre o tema.

Espera-se com este trabalho, que a prática da intervenção Relaxamento com Imagem Guiada seja divulgada e difundida no trabalho do dia-a-dia do enfermeiro, que possa em um futuro próximo oferecer a intervenção não somente ao paciente com câncer, em tratamento quimioterápico, mas 
também a outros pacientes e em outros tratamentos.

\section{REFERÊNCIAS}

1. Gaston-Johansson F, Fall-Dickson JM, Nanda JP, Sarenmalm EK, Browall M, Goldstein F. Long-term effect of the self-management comprehensive coping strategy program on quality of life in patients with breast cancer treated with high-dose chemotherapy. Psychooncology. [Internet] 2013;22(3) [acesso em 14 mar 2016]. Disponível: http:// dx.doi.org/10.1002/pon.3031.

2. Syrjala KL, Jensen MP, Mendoza ME, Yi JC, Fisher HM, Keefe FJ. Psychological and behavioral approaches to cancer pain management. J Clin Oncol. [Internet] 2014;32(16) [acesso em 14 mar 2016]. Disponível: http://jco. ascopubs.org/content/early/2014/05/05/JCO.2013.54.4825.

3. Deep CN, Leal I, Patrão I. Avaliação da intervenção cognitivo-comportamental em gestão do stress em pacientes com fadiga oncológica, em radioterapia. Saúde soc. [Internet] 2014;23(1) [acesso em 14 mar 2016]. Disponível: http://dx.doi.org/10.1590/S0104-12902014000100023.

4. Stommel M, Kurtz ME, Kurtz JC, Given CW, Given BA. A longitudinal analysis of the course of depressive symptomatology in geriatric patients with cancer of the breast, colon, lung, or prostate. Health Psychol. [Internet] 2004;23(6) [acesso em 25 jan 2016]. Disponível: http://dx.doi.org/10.1037/0278-6133.23.6.564.

5. de Souza BF, Pires FH, Dewulf NLS, Inocenti A, Silva AEBC, Miasso AI. Pacientes em uso de quimioterápicos: depressão e adesão ao tratamento. Rev. esc. enferm. USP. [Internet] 2013;47(1) [acesso em 14 mar 2016]. Disponível: http://dx.doi.org/10.1590/S0080-62342013000100008.

6. Zhou K, Li X, Li J, Liu M, Dang S, Wang D, et al. A clinical randomized controlled trial of music therapy and progressive muscle relaxation training in female breast cancer patients after radical mastectomy: Results on depression, anxiety and length of hospital stay. Eur J Oncol Nurs. [Internet] 2015;19(1) [acesso em 14 mar 2016]. Disponível: http://dx.doi.org/10.1016/j.ejon.2014.07.010.

7. Witer-Janusek L, Albuquerque K, Chroniak KR, Chroniak C, Durazo-Arvizu R, Mathews HL. Effect of mindfulness based stress reduction on immune function, quality of life and coping in women newly diagnosed with early stage breast cancer. Brain Behav Immun. [Internet] 2008;22(6) [acesso em 25 jan 2016]. Disponível: http://dx.doi. org/10.1016/j.bbi.2008.01.012.

8. Hoon LS, Sally CWC, Hong-Gu H. Effect of psychosocial interventions on outcomes of patients with colorectal cancer: a review of the literature. Eur J Oncol Nurs. [Internet] 2013;17(6) [acesso em 14 mar 2016]. Disponível: http://dx.doi.org/10.1016/j.ejon.2013.05.001.

9. Hart LK, Freel MI, Haylock PJ, Lutgendorf SK. The use of healing touch in integrative oncology. Clin J Oncol Nurs. [Internet] 2011;15(5) [acesso em 14 mar 2016]. Disponível: http://dx.doi.org/10.1188/11.CJON.519-525.

10. Serra D, Parris CR, Carper E, Homel P, Fleishman SB, Harrison LB, et al. Outcomes of guided imagery in patients receiving radiation therapy for breast cancer. Clin J Oncol Nurs. [Internet] 2012;16(6) [acesso em 14 mar 2016]. Disponível: http://dx.doi.org/10.1188/12.CJON.617-623.

11. Chralambous A. P101 A randomized trial of the effect of training in progressive muscle relaxation and guidedimagery techniques in improving psychological and quality-of-life for breast and prostate cancer patients receiving chemotherapy. Eur J Oncol Nurs. [Internet] 2010;14(Suppl. 1) [acesso em 14 mar 2016]. Disponível: http://dx.doi.org/10.1016/S1462-3889(10)70163-0.

12. Gordon JS. Mind-body medicine and cancer. Hematol Oncol Clin N Am. [Internet] 2008;22(4) [acesso em 25 jan 2016]. Disponível: http://dx.doi.org/10.1016/j.hoc.2008.04.010.

13. Pert C. Conexão mente corpo espírito para o seu bem-estar: uma cientista ousada avalia a medicina alternativa. Pert C, Marriot N, organizadoras. Traduzido por Júlia Bárány Yaari. São Paulo: ProLíbera Editora; 2009.

14. Carlson LE, Bultz BD. Mind-body interventions in oncology. Curr Treat Options Oncol. [Internet] 2008;9(2-3) [acesso em 25 jan 2016]. Disponível: http://dx.doi.org/10.1007/s11864-008-0064-2.

15. Polit DF, Beck CT, Hungler BP. Fundamentos de pesquisa em enfermagem: métodos, avaliação e utilização. $5^{a}$ 
ed. Porto Alegre: Artmed; 2004.

16. Sawada NO, Zago MMF, Galvão CM, Cardozo FMC, Zandonai AP, Okino L, et al. The outcomes of visualization and acupuncture on the quality of life of adult cancer patients receiving chemotherapy. Cancer Nurs. [Internet] 2010;33(5) [acesso em 25 jan 2016]. Disponível: http://dx.doi.org/10.1097/NCC.0b013e3181d86739.

17. Gorestein C, Andrade L. Validation of Portuguese version of Beck depression inventory and state-trait anxiety inventory in Brazilian subjects. Braz J Med Biol Res. 1996;29(4):453-7.

18. de Paula JM, Sonobe HM, Nicolussi AC, Zago MMF, Sawada NO. Symptoms of depression in patients with cancer of the head and neck undergoing radiotherapy treatment: a prospective study. Rev. Latino-Am Enfermagem. [Internet] 2012;20(2) [acesso em 25 jan 2016]. Disponível: http://dx.doi.org/10.1590/S0104-11692012000200020.

19. Ministério da Saúde (BR). Instituto Nacional de Câncer José Alencar Gomes da Silva (INCA). Coordenação de Prevenção e Vigilância. Estimativa 2016: incidência de câncer no Brasil/Instituto Nacional de Câncer José Alencar Gomes da Silva. Rio de Janeiro: INCA; 2015.

20. Zhang M, Chan SW-C, You L, Wen Y, Peng L, Liu W, et al. The effectiveness of a self-efficacy-enhancing intervention for Chinese patients with colorectal cancer: A randomized controlled trial with 6-month follow up. Int J Nurs Studies. [Internet] 2014;51(8) [acesso em 14 mar 2016]. Disponível: http://dx.doi.org/10.1016/j. ijnurstu.2013.12.005.

21. Wyatt G, Sikorskii A, Siddiqi A, Given CW. Feasibility of a reflexology and guided imagery intervention during chemotherapy: results of a quasi-experimental study. Oncol Nurs Forum. [Internet] 2007;34(3) [acesso em 25 jan 2016]. Disponível: http://dx.doi.org/10.1188/07.ONF.635-642. 